\title{
Mapping Evidence on the Management of Diabetic Retinopathy Using Teleophthalmology to Complement Human Resources for Eye Health: A Scoping Review Protocol
}

\author{
Thembile Zikhali ${ }^{1} \&$ Zamadonda Nokuthula Xulu-Kasaba ${ }^{1}$ \\ ${ }^{1}$ Department of Optometry, School of Health Sciences, University of KwaZulu-Natal, Westville Campus, Durban, \\ South Africa \\ Correspondence: Thembile Zikhali, University of KwaZulu-Natal, School of Health Sciences, Westville Campus, \\ E-Block, Durban, 4000, South Africa. Tel: 27-82-079-2594. E-mail: zicallism@gmail.com
}

Received: December 4, 2019 Accepted: April 20, 2020 Online Published: May 8, 2020

doi:10.5539/gjhs.v12n7p11 URL: https://doi.org/10.5539/gjhs.v12n7p11

\begin{abstract}
Introduction: Diabetic Retinopathy is the leading cause of blindness in working adults, and remains a serious public health concern, globally. Whilst extensive research has been done in this area, the prevention of diabetic retinopathy is still very poor. Attributing factors in previous studies have included shortage of Human Resources for Eye Health in primary health centres, and a shortage of technology and skill in most countries. In some countries, teleophthalmology has been introduced to assist in the management of diabetic retinopathy by capacitating Eye Health professionals in primary health care settings since diabetes is diagnosed at this level. Where implemented, management of diabetic retinopathy through teleophthalmology has further contributed to reduction in preventable blindness while minimizing turnaround times as patients no longer have to wait for an Ophthalmologist appointment. The main objective of this scoping review is to map evidence on the use of teleophthalmology in the management of diabetic retinopathy globally.
\end{abstract}

Methods and Analysis: Methodology will be guided by Arksey and O'Malley scoping review framework. We will search for literature from the following EBSCO Host databases: MEDLINE, Health source: Nursing/Academic Edition, Health source - Consumer, CINAHL and Academic Search Complete. PubMed, Google Scholar and Science Direct will also be searched for studies. Where articles are not accessible online, the author will be contacted for the article, failing which the University of KwaZulu-Natal library services will be asked for assistance. The literature search will be done for the period from January 2014 to October 2019 where all studies in English will be accepted. After the initial search, title, abstract and full text screening will be conducted by two independent reviewers. To assess the methodological rigour of included studies, the Mixed Method Appraisal tool (MMAT) 2018 will be used. Study results will be presented using Preferred Reporting Items for Systematic Reviews and Meta-Analyses (PRISMA) chart.

Discussion: It is anticipated that this scoping review will identify suitable literature on diabetic retinopathy and its management through teleophthalmology. The findings of this review will be disseminated through peer-reviewed journals and information summarized will be used to guide future research.

Keywords: Diabetic retinopathy, teleophthalmology, telemedicine, Low-to-middle-income countries, Diabetic Eye Disease

\section{Introduction}

Diabetic retinopathy is the main cause of new onset blindness in many developed countries (Organization, 2006), and a common cause of blindness in many low-to-middle-income (LMIC) countries (Klein et al., 1992). Named one of the World Health Organisation's (WHO) priority eye diseases leading to preventable visual impairment and blindness, diabetic retinopathy (DR) is a vascular disease of the retina which affects patients with long-standing diabetes (Organization). Due to the high blood glucose levels in diabetics' bloodstream, damage is caused to the blood vessels in the retina causing diabetic retinopathy, which further results to diabetic macula oedema, and ultimately blindness (Organization, 2006). With a global estimate of 652.1 million in 2016 to a predicted 1.42 billion by 2045 (Cho et al., 2018), the WHO estimated that the number of people with DR who will progress to 
vision-threatening diabetic retinopathy (VTDR) will increase from 37.3 million to 56.3 million in 2030, making diabetic retinopathy the leading cause of new-onset blindness in industrialised countries, and a growing concern in middle income countries (Organization, 2006).

The reality of the insidious onset of Type 2 diabetes results in many patients remaining undiagnosed for years and presenting with DR at the time of diagnosis (Cook, 2014). Type 1 diabetics, on the other hand, are diagnosed early in the course of their disease, and they typically do not develop retinopathy until years after the diagnosis is made. One of methods used to diagnose DR is teleophthalmology. This is an area of telemedicine, where digital images from a patient's eye are taken by non-ophthalmologists and forwarded to Ophthalmology professionals for expert guidance on interventions and patient management (Sreelatha \& Ramesh, 2016). With a severe shortage of Human Resources for eye Health (HReH), especially in Low-to-Middle income countries (LMIC) such as India, this method of intervention has largely relieved the eye health system. Not only has teleophthalmology reduced waiting periods and delays in the referral system, reduced costs it has further ensured that tests are not repeated, and interventions for ophthalmology-related conditions are implemented earlier (Grisolia et al., 2017).

Tele-ophthalmology has been utilised in many areas to successfully improve the reach of specialist services (Prathiba \& Rema, 2011) whilst improving rate of patient screening, turnaround times for retinal disease management and access to eye health for those in remote areas (Gupta et al., 2017), ultimately resulting in the prevention of vision loss overall (Silva et al., 2016). Various reviews have been conducted to ascertain the accuracy, specificity and reliability of teleophthalmology in general eye conditions including Retinopathy of Prematurity, neuroophthalmology, glaucoma and diabetic retinopathy (Whited, 2006; Sreelatha \& Ramesh, 2016; Bahaadinbeigy \& Yogesan, 2011).

This scoping review will specifically focus on literature pertaining screening and management of diabetic retinopathy through teleophthalmology. Interventions used to facilitate screening practices will emerge and assist in screening strategies that enable an earlier detection of DR. The aim of the study is to map evidenced information on the use of Teleophthalmology in the management of diabetic retinopathy. It is hoped that this literature will help with exploring strategies that will enable earlier disease diagnosis, management and treatment in order to avoid unnecessary vision loss from untreated diabetic retinopathy. It is further anticipated that the emerging literature will highlight research gaps to guide future research in this area.

\section{Methods}

This systematic scoping review will be guided by the Arksey and O'Malley scoping review framework (Arksey \& O'Malley, 2005) as per the Joanna Briggs Institute manual (Peters et al., 2015). The following 5 stages will be followed in the study:

1. Identifying the search question

2. Identifying relevant studies

3. Study selection

4. Charting the data

5. Collating, summarizing and reporting the results.

A quality assessment of the included studies will be conducted in this scoping review, to assess methodology of included studies, following recommendations by Levac et al. (Levac et al., 2010).

\subsection{Identifying the Research Question}

The main research question is: What is the available evidence on the use of teleophthalmology in the early management of diabetic retinopathy?

\subsection{Eligibility of the Research Question}

The study will employ the Population, Concept and Context (PCC) framework to determine eligible studies that respond to the research question. The framework has been shown in Table 1. 
Table 1. PCC model

\begin{tabular}{ll}
\hline Population & $\begin{array}{l}\text { Diabetic patients, Ocular manifestations of diabetes, Ocular complications of diabetes, diabetic } \\
\text { retinopathy, retinal complications of diabetes. }\end{array}$ \\
\hline Concept & Tele-ophthalmology or Telemedicine \\
\hline Context & All available studies (globally) \\
\hline
\end{tabular}

\subsection{Identifying Relevant Studies}

Completed primary studies will be searched for using the following databases: PubMed, Google Scholar, Science Direct and EBSCO Host databases MEDLINE, Health source: Nursing/Academic Edition, Health source Consumer, CINAHL and Academic Search Complete. Qualitative, quantitative and mixed method studies, published in English, will be included in the scoping review. The search will be carried out using keywords as well as Medical Subject Headings (MeSH) terms and Boolean terms. "AND" will be used when separating concepts, whilst "OR" will be used to separate synonyms.

To evaluate feasibility, a pilot study was conducted using the following search terms:

Search: Diabetes mellitus OR diabetic AND ocular manifestations OR ocular complications OR retinopathy OR retinal diseases AND Telemedicine OR Teleophthalmology.

\subsection{Study Selection}

In order to answer the research question, and select only the relevant studies, careful study selection is required. To ensure this, various inclusion and exclusion criteria will be adhered to.

\subsubsection{Inclusion Criteria}

Studies meeting the following criteria will be included:

- All studies available from 2014 - 2019

- Studies on diabetic human subjects with no gender restriction

- Studies on ocular manifestations of diabetes or diabetic retinopathy and telemedicine or teleophthalmology

\subsubsection{Exclusion Criteria}

The following exclusion criteria will be used:

- All studies from prior to 2014

- Studies on animal subjects

- Studies on non-diabetic subjects

- Studies that are not primary studies eg review articles

- Studies that only show evidence on one phenomenon and not both diabetic retinopathy and teleophthalmology

Table 2. Pilot study search results

\begin{tabular}{ll}
\hline Date of search & 8 October 2019 \\
\hline Keywords used & $\begin{array}{l}\text { diabetes mellitus OR diabetes AND ocular manifestations OR ocular complications OR } \\
\text { retinopathy OR retinal disease AND telemedicine OR teleophthalmology }\end{array}$ \\
\hline Period searched & $01 / 01 / 2014-08 / 10 / 2019$ \\
\hline Search engine & Retrieved Sources \\
\hline Pubmed & 393 \\
\hline Google scholar & 3050 \\
\hline EBSCOhost & 217046 \\
\hline Science Direct & 144028 \\
\hline
\end{tabular}


Study selection will follow thorough screening by two independent screeners, using a three-stage strategy. The Primary investigator (PI) will create screening tools for title, abstract and full text screening using Google forms. All tools will be piloted prior to being used.

The PI will initially search for studies and store them in a library in Endnote $\mathrm{x} 8$ created especially for this study. Thereafter, duplicates will be removed and screening will begin.

Title screening: The PI will conduct this alone to ensure that the selected studies are all in the area of interest. After finalising this, the endnote library with then be shared with the second reviewer.

Abstract screening: Adhering to specified inclusion and exclusion criteria, two independent reviewers will conduct abstract screening using piloted tools. All relevant articles that adhere to stipulated criteria. On agreement of the final articles, the next step follows.

Full text screening: Using the piloted tools to exclude articles not meeting the inclusion criteria, both independent screeners will conduct full article screening in order to decide on the final articles to be included in the study. Where the full article cannot be found online, University of KwaZulu Natal (UKZN) library services will also be used to find articles that are needed or contact authors to request full articles. Where full articles cannot be found despite all attempts to obtain them, the affected studies will be excluded. If there is any discrepancy between the reviewers, this will be resolved by a discussion, and the final articles included.

Screening results will be recorded using an adapted Preferred Reporting Items for Systematic Review and Meta-Analyses (PRISMA) guideline (Moher et al., 2009), summarised in Figure 1. 


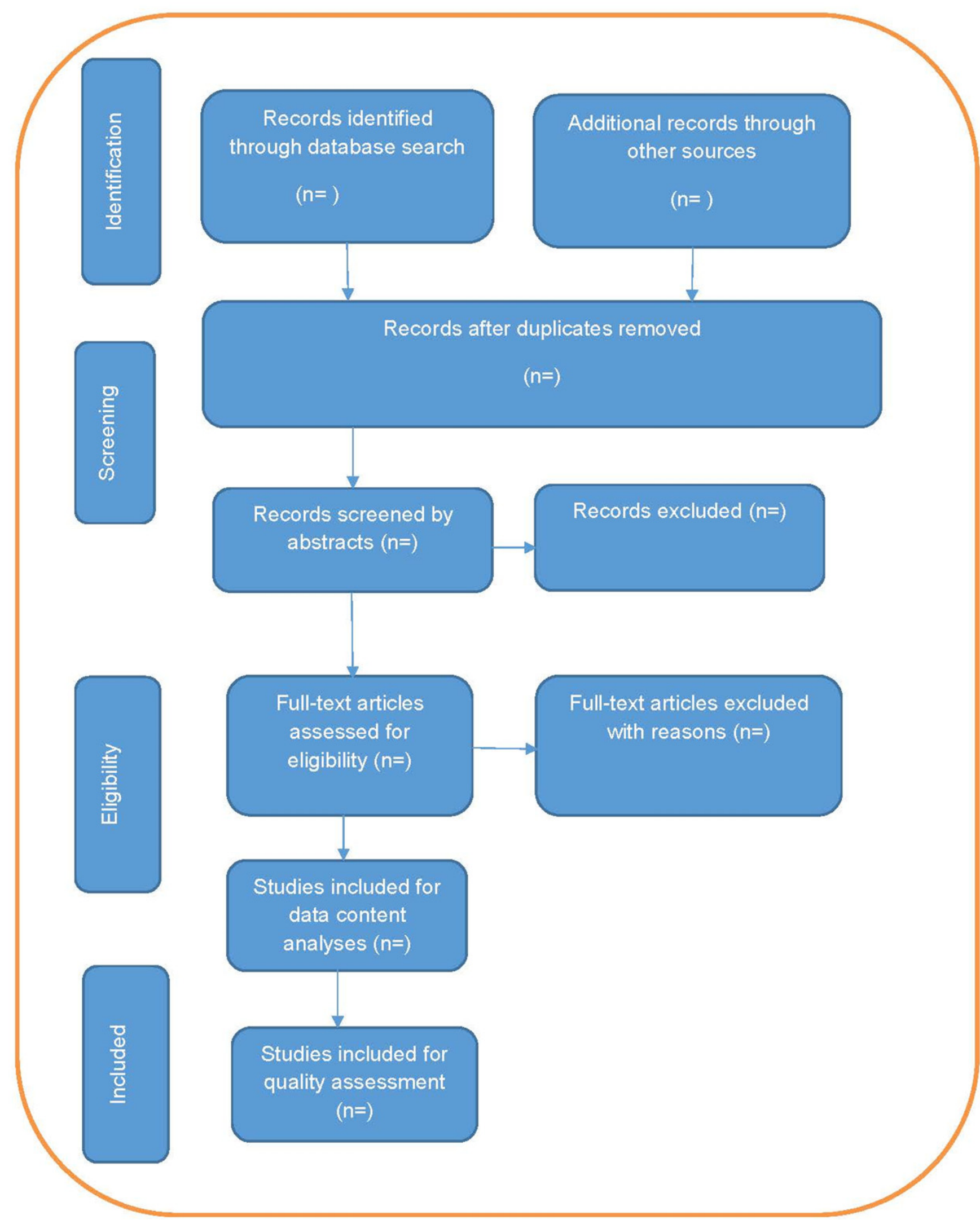

Figure 1. Flow chart showing literature search and selection of studies. 


\subsection{Data Charting}

A data charting tool (Table 3) will be formulated and piloted prior to being used. This tool will be piloted by two independent reviewers on 5 randomly sampled, included articles to ensure consistency with the study. The tool will then be modified where necessary, following this exercise. Data charting will then be effected. Where updating becomes necessary during data charting, this will be done frequently. Data will be categorised and arranged to classify the various outcomes and themes to be analysed from the included studies. Extracted data will also be recorded on the data extraction tool.

\subsection{Collating, Summarizing and Reporting of Results}

As the aim of this study is to map evidence on the use of teleophthalmology in managing diabetic retinopathy, data extracted should be in line with the areas of interest. Thematic content analysis from the extracted data will look at a few pivotal areas in the use of teleophthalmology. Themes to be explored will be focused along the following outcomes: diagnostic accuracy of teleophthalmology, effectiveness of DR screening using specific features in a camera, and teleophthalmology increasing the rate of DR evaluation in rural settings where there is a shortage of human resources for eye health.

Emerging and resulting themes will then be analysed thoroughly to determine their relevance and response to the research question. This will all be done with a careful cognisance of the aim of the study to ensure that outcomes and results adhere to the main aim, whilst also exposing all gaps in the existing pool of knowledge, existing policies and current practices.

\subsection{Quality Appraisal}

The Levac et al recommendations for quality appraisal of included studies would be performed by two reviewers (Levac et al., 2010). The Mixed Method Appraisal Tool (MMAT) version 2018 is preferred as it is able to assess all common types of study methodologies and design (Hong et al., 2018). Quality assessment will look at the aim of each study, methodology, study design, and participant adequacy in each study. Appraisal of studies will include an actual scoring of study quality, ranging from $25 \%$ for low quality $100 \%$ for areas in the study that score well, following the guidance of the MMAT tool.

Table 3. Data Extraction table

\begin{tabular}{l}
\hline Author and Date \\
\hline Study title \\
Study design \\
Country income classification \\
Mean age of participants \\
Duration of condition \\
Digital device used \\
Who takes the picture \\
Technology used to forward picture (e.g. email) \\
Who interprets the picture \\
Type of program used \\
Mydriatic used \\
Intervention strategy communicated \\
Turnaround time \\
Other systemic diseases \\
Other complications \\
Emerging themes \\
\hline Significant findings
\end{tabular}




\section{Discussion}

With a severe shortage of Human Resources for eye Health (HReH) found in the SSA (Palmer et al., 2014), teleophthalmology was introduced in rural health centres to improve quality of medical services and outreach. This was done with the aim to improve services in rural areas to that of services in urban areas (Prathiba \& Rema, 2011). Patient screening rate and satisfaction has been improved through the incorporation of teleophthalmological screening, using various digital gadgets to capture retinal pictures, and send them to an Ophthalmologist to identify DR and prevent vision loss (Silva et al., 2016). The introduction of teleophthalmology in India has resulted in increased access to eye health in remote areas (Gupta et al., 2017).

As diabetic retinopathy continues to emerge as one of the leading causes of visual impairment and blindness globally (Mabaso, 2008), VISION 2020: Right to Sight goals for reducing preventable blindness need to be evaluated at a local level. As a priority disease, and the third leading cause of preventable blindness in South Africa (Mabaso \& Oduntan, 2014), it is vital that earlier screening be prioritised, and earlier interventions be made for patients to be diagnosed prior to complications and development of the disease. Tele-ophthalmology has proven successful in narrowing this gap and enabling earlier diagnosis and management of diabetic retinopathy (Hautala et al., 2014). Earlier interventions and diagnosis at Primary Health Level ensure that the patient is assisted holistically at one clinic visit. It also ensures that Ophthalmology involvement is accelerated, and blindness prevented much earlier in the disease.

\section{Conclusion}

It is envisaged that this scoping review will help to identify from the existing literature and cost effective methods that could be introduced at primary health care level to reduce the burden of avoidable blindness secondary to DR. According to the authors' knowledge no previous scoping reviews on this matter have been done in South Africa or Africa. The result of this review will be useful in determining areas needing further research. This study may pave a way towards improving the quality of eye care services at PHC level and it may add to an enhanced diabetic retinopathy screening and management system for LMICs.

\subsection{Ethics and Dissemination}

No ethical approval is required as the review will be done on published data. The results of the study will published through peer-reviewed journals. All data utilised is already available in the public domain.

\section{Acronyms}

PI: Primary Investigator

RE: Refractive error

DR: diabetic retinopathy

HReH: Human Resources for Eye Health

LMIC: Low-to-middle-income countries

SSA: Sub-Saharan Africa

UKZN: University of KwaZulu-Natal

\section{Acknowledgements}

The Authors would like to acknowledge Mbuzeni Hlongwa and Mumbi Chola for reviewing this manuscript.

\section{Authors' Contributions}

TZ and ZXK conceptualized the study. TZ prepared the manuscript under the guidance and supervision of ZXK. Both authors contributed to the development and design of the study. TZ drafted the manuscript and ZXK reviewed it. Both authors contributed to the final version of the manuscript and agree to publication thereof.

\section{Competing Interests Statement}

The authors declare that there are no competing or potential conflicts of interest.

\section{References}

Arksey, H., \& O'malley, L. (2005). Scoping studies: towards a methodological framework. International journal of social research methodology, 8, 19-32. https://doi.org/10.1080/1364557032000119616

Bahaadinbeigy, K., \& Yogesan, K. (2011). Advances in teleophthalmology: Summarising published papers on teleophthalmology projects. Advances in telemedicine: Applications in various medical disciplines and 
geographical regions. Rijeka, Croatia: InTech Open, 231-242. https://doi.org/10.5772/13595

Cho, N., Shaw, J., Karuranga, S., Huang, Y., Da Rocha Fernandes, J., Ohlrogge, A., \& Malanda, B. (2018). IDF Diabetes Atlas: Global estimates of diabetes prevalence for 2017 and projections for 2045. Diabetes research and clinical practice, 138, 271-281. https://doi.org/10.1016/j.diabres.2018.02.023

Cook, S. (2014). The South African diabetic retinopathy screening programme launches: diabetes news. South African Journal of Diabetes and Vascular Disease, 11, 96. https://doi.org/10.4172/2155-6156.1000359

Grisolia, A. B. D., Abalem, M. F., Lu, Y., Aoki, L., \& Matayoshi, S. (2017). Teleophthalmology: where are we now? Arquivos brasileiros de oftalmologia, 80, 401-406. https://doi.org/10.5935/0004-2749.20170099

Gupta, A., Cavallerano, J., Sun, J. K., \& Silva, P. S. (2017). Evidence for telemedicine for diabetic retinal disease. Seminars in ophthalmology, 2017, 22-28. https://doi.org/10.1080/08820538.2016.1228403

Hautala, N., Aikkila, R., Korpelainen, J., Keskitalo, A., Kurikka, A., Falck, A., Bloigu, R., \& Alanko, H. (2014). Marked reductions in visual impairment due to diabetic retinopathy achieved by efficient screening and timely treatment. Acta ophthalmologica, 92, 582-587. https://doi.org/10.1111/aos.12278

Hong, Q. N., Fàbregues, S., Bartlett, G., Boardman, F., Cargo, M., Dagenais, P., ... \& O'cathain, A. (2018). The Mixed Methods Appraisal Tool (MMAT) version 2018 for information professionals and researchers. Education for Information, 34, 285- 291. https://doi.org/10.3233/EFI-180221

Klein, R., Klein, B. E., Moss, S. E., \& Linton, K. L. (1992). The Beaver Dam Eye Study: retinopathy in adults with newly discovered and previously diagnosed diabetes mellitus. Ophthalmology, 99, 58-62. https://doi.org/10.1016/S0161-6420(92)32011-1

Levac, D., Colquhoun, H., \& O'brien, K. K. (2010). Scoping studies: advancing the methodology. Implementation science, 5, 69. https://doi.org/10.1186/1748-5908-5-69

Mabaso, R. (2008). Blindness and visual impairment among people with diabetes mellitus 40 years and older in the Limpopo Province, South Africa.

Mabaso, R. G., \& Oduntan, O. A. (2014). Risk factors for visual impairment and blindness amongst black adult diabetics receiving treatment at Government healthcare facilities in Mopani District, Limpopo province, South Africa. African journal of primary health care \& family medicine, 6, 1-8. https://doi.org/10.4102/phcfm.v6i1.623

Moher, D., Liberati, A., Tetzlaff, J., \& Altman, D. G. (2009). Preferred reporting items for systematic reviews and meta-analyses: the PRISMA statement. BMJ, 339. https://doi.org/10.1136/bmj.b2535

Organization, W. H. (2006). International Agency for the Prevention of Blindness. Global Action Plan 20142019.

Organization, W. H. (2006). Prevention of blindness from diabetes mellitus: report of a WHO consultation in Geneva, Switzerland, 9-11 November 2005, World Health Organization.

Palmer, J. J., Chinanayi, F., Gilbert, A., Pillay, D., Fox, S., Jaggernath, J., .. \& Blanchet, K. (2014). Mapping human resources for eye health in 21 countries of sub- Saharan Africa: current progress towards VISION 2020. Human resources for health, 12, 44. https://doi.org/10.1186/1478-4491-12-44

Peters, M., Godfrey, C., Mcinerney, P., Soares, C. B., Khalil, H., \& Parker, D. (2015). Methodology for JBI scoping reviews. The Joanna Briggs Institute Reviewers manual 2015. The Joanna Briggs Institute.

Prathiba, V., \& Rema, M. (2011). Teleophthalmology: a model for eye care delivery in rural and underserved areas of India. International journal of family medicine, 2011. https://doi.org/10.1155/2011/683267

Silva, P. S., Horton, M. B., Clary, D., Lewis, D. G., Sun, J. K., Cavallerano, J. D., \& Aiello, L. P. (2016). Identification of diabetic retinopathy and ungradable image rate with ultrawide field imaging in a national teleophthalmology program. Ophthalmology, 123, 1360-1367. https://doi.org/10.1016/j.ophtha.2016.01.043

Sreelatha, O. K., \& Ramesh, S. V. (2016). Teleophthalmology: improving patient outcomes? Clinical ophthalmology (Auckland, NZ), 10, 285. https://doi.org/10.2147/OPTH.S80487

Whited, J. D. (2006). Accuracy and reliability of teleophthalmology for diagnosing diabetic retinopathy and macular edema: a review of the literature. Diabetes technology \& therapeutics, 8, 102-111. https://doi.org/10.1089/dia.2006.8.102 


\section{Copyrights}

Copyright for this article is retained by the author(s), with first publication rights granted to the journal.

This is an open-access article distributed under the terms and conditions of the Creative Commons Attribution license (http://creativecommons.org/licenses/by/4.0/). 